\title{
Genome-Wide Discovery of cis-Elements in Promoter Sequences Using Gene Expression
}

\author{
Maxim Troukhan, ${ }^{1}$ Tatiana Tatarinova,${ }^{2}$ John Bouck, ${ }^{1}$ Richard B. Flavell, ${ }^{1}$ and Nickolai N. Alexandrov ${ }^{1}$
}

\begin{abstract}
The availability of complete or nearly complete genome sequences, a large number of $5^{\prime}$ expressed sequence tags, and significant public expression data allow for a more accurate identification of cis-elements regulating gene expression. We have implemented a global approach that takes advantage of available expression data, genomic sequences, and transcript information to predict cis-elements associated with specific expression patterns. The key components of our approach are: (1) precise identification of transcription start sites, (2) specific locations of cis-elements relative to the transcription start site, and (3) assessment of statistical significance for all sequence motifs. By applying our method to promoters of Arabidopsis thaliana and Mus musculus, we have identified motifs that affect gene expression under specific environmental conditions or in certain tissues. We also found that the presence of the TATA box is associated with increased variability of gene expression. Strong correlation between our results and experimentally determined motifs shows that the method is capable of predicting new functionally important cis-elements in promoter sequences.
\end{abstract}

\section{Introduction}

$\mathbf{T}$ HE AMOUNT OF GENETIC INFORMATION available for computational analysis continues to increase: from whole genome sequences, to expressed sequence tags (ESTs) and to expression data. The GenBank resource at the National Center for Biotechnology Information grows at an exponential rate (Benson et al., 2007; NCBI Press Release, 2005). The challenge for computational biologists today is how to extract new knowledge from the large and diverse data sets. The data available to researchers is also of variable quality. ESTs represent a good example of a data set that has several caveats, such as variable quality of libraries and sequences. However, the volume of data available through EST sequencing efforts allows useful information to be extracted.

Ever since transcription factors were first shown to bind to upstream regions, there has been a challenge to identify cis-elements that contribute to the expression level of an adjacent gene (Argüello-Astorga and Herrera-Estrella, 1998; Suzuki et al., 1995). Early on, this was accomplished by extracting conserved elements from aligned upstream regions of genes that were expressed under the same physiological condition (Bailey and Elkan, 1994; Galas et al., 1985; Ghosh and Echols, 1972; Hertz and Stormo, 1999; Hughes and Estep, 2000; Roth et al., 1998). Several important cis-elements have been identified through this approach (Meyersa et al., 2003). As information from molecular experiments became available, additional resources have been built upon the curation and computational extension of these, with the most familiar being TRANSFAC (Wingender et al., 2001). A recent benchmark of motif-finding programs has concluded that motif identification "remains a wonderful and complex challenge for computational biologists" (Tompa et al., 2005). The search for biologically important cis-elements can be made more reliable by increasing the number of analyzed promoters. However, NP-completeness of a multiple alignment problem makes it difficult to analyze a large number of long promoter sequences in realistic time.

Microarray data provide measurements of the transcript abundance and the implicit assumption is often made that it reflects promoter activity. However, this assumption may not always be correct, because mRNA levels are also affected by transcript stability or different cell types represented in a sample. This effect might decrease accuracy of promoter motif finding methods that rely upon preselection of a set of similarly expressed genes. However, genome-wide methods

${ }^{1}$ Ceres, Inc. 1535 Rancho Conejo Road, Thousand Oaks, California 91310

2Department of Mathematics, Loyola Marymount University, Los Angeles, California 90045.

The first two authors contributed equally to this article. 
that are probabilistic in nature will have higher confidence scores and will be able to identify weaker motif associations. One such strategy was developed in a series of publications by (Foat et al., 2006; Foat et al., 2005) through which they developed the REDUCE algorithm, employing multivariate regression on combined expression and promoter data sets. Both REDUCE and the latest versions MatrixREDUCE are powerful algorithms that are able to find significant associations between potential cis-elements and microarray expression. MatrixREDUCE infers the sequence specificity of a Transcription Factor (TF) from genome-wide occupancy data by fitting a statistical mechanical model for TF-DNA interaction (Foat et al., 2005).

Many of the promoter motif-finding methods rely on correct identification of upstream regions and Transcription Start Sites (TSS). It was previously reported (Berendzen et al., 2006; Pritsker et al., 2004) that the position of the transcription factor binding site with respect to the TSS plays a key role in the specific programming of regulatory logic within noncoding regions, highlighting the importance of determining the precise location of TSS for motif discovery. A simple approach is to use a collection of $5^{\prime}$ ESTs to predict the position of TSS. One of the problems with the $5^{\prime}$ ESTs is that even in the best libraries, only $50-80 \%$ of the $5^{\prime}$ ESTs extend to the TSSs (Ohler, 2002; Sugahara et al., 2001; Suzuki et al., 1997). The traditional approach to predict the position of the TSS was based on finding the position of the longest $5^{\prime}$ transcripts (for overview of eukaryotic promoter prediction methods see Fickett and Hatzigeorgiou, 1997). Recently, it has been demonstrated that the quality of TSS prediction can be improved when combining data from multiple sources, such as collections of $5^{\prime}$ EST and conserved DNA sequence motifs (Ohler, 2006; Ohler et al., 2002). In 2002, Down and Hubbard developed a machine-learning approach (Eponine) to build useful models of promoters; their method uses weight matrices for the most significant motifs around the TSS (e.g., TATA box and CpG island) to predict the position of the TSS. Eponine was tested on human chromosome 22 and detected $>50 \%$ of experimentally validated transcription start sites, but on some occasions it did not predict the direction of transcription correctly. Recently, Abeel et al. (2008) presented EP3, promoter prediction method based on the structural large-scale features of DNA, including bendability, nucleosome position, free energy, and protein-DNA twist. EP3 identifies the region on a chromosome that is likely to contain TSS, but it does not predict the direction of a promoter. We propose a different method of TSS prediction that considers positional frequency of $5^{\prime}$ EST matches on genomic DNA together with the gene model and allows an accurate determination of the TSS.

Extending from accurate TSS identification we also describe a new statistical approach for cis-element identification that combines gene expression with promoter sequence information. We apply this method to the promoters of the model plant Arabidopsis thaliana that contains 27,000 genes (Initiative, 2000; Yamada et al., 2003). Approximately 22,000 of them have 5'-FL cDNA sequences available for TSS identification (Alexandrov et al., 2006). Expression of $\sim 22,000$ Arabidopsis genes represented on Affymetrix ATH1 chip has been measured by many research groups in various tissues and experimental conditions. Over 2,000 ATH1 hybridization slides are publicly available (e.g., from NASC database:
http://Arabidopsis.info/). We have combined expression data with promoter sequences to identify motifs associated with expression pattern of interest. Our method provides an estimate of the statistical significance of the motif and identifies motifs which are responsible for gene activation as well as for transcription repression. Importantly, this method does not require preselection of the coregulated set of genes; it is also computationally efficient and applicable to any expression pattern.

We employed our methods to find motifs associated with stress induction, tissue-specific expression, overall promoter strength, and expression variability. Drought and seed-specific motifs identified through these methods show strong correlation with motifs that have been experimentally validated. We have also found motifs that affect general promoter strength and provide additional insight into the function of the well characterized TATA box.

\section{Materials and Methods}

\section{Transcription start site finding algorithm (TSSer)}

For our analysis we used 188,506 5' EST sequences and 25,026 full-length mRNA sequences of Arabidopsis thaliana, available at GenBank. We have employed The Arabidopsis Information Resource (TAIR) genomic annotation for Arabidopsis thaliana (released Sept, 2007). We have developed a new algorithm, called TSSer, to reliably predict positions of TSSs. Briefly, we align EST and mRNA sequences against the corresponding genomic sequence using Washington University Blast. Alignments are further refined and extended as described in (Alexandrov et al., 2006). If multiple loci per EST or mRNA occur, we select the best one based on the identity of the match to the genomic reference. For each 5' EST the start of the alignment to the genome is set as a putative TSS. Counts of putative TSSs in the sliding window of size $\mathrm{W}$ are recorded. The most frequent position of the most populated window that is not located inside the coding region for the locus is designated as the best TSS. Choice of the window size depends on the quality of the genome and EST collection. Default value of W, used to annotate Arabidopsis thaliana promoters, is one nucleotide. Using this technique we have predicted positions of TSSs for 15,853 loci $(13,279$ of which contain at least two confirming ESTs) in the Arabidopsis thaliana genome. Examination of the position of known promoter motifs from TRANSFAC database indicated that the vast majority of these were found within the region 300 nucleotides upstream of the TSS to 50 bases downstream (similar conclusions were also reached by Berendzen et al., 2006). Based upon this, for further analysis we used promoter sequences 300 nucleotides upstream from the TSS and 100 nucleotides downstream. We have compared promoters predicted by TSSer with promoters predicted by The Arabidopsis Information Resource (TAIR), publicly available at ftp://ftp.Arabidopsis.org/home/tair/seq_analysis_ updates/. They use the Sim4 (Florea, 1998) and BLAT (Kent, 2002) algorithms to align all cDNAs and ESTs available from GenBank to the genome. These aligned sequences are then assembled into gene models using PASA (Haas et al., 2003). TAIR assigns one TSS to each gene model, typically the one resulting in the longest ORF; therefore, their TSS annotations are based on the longest full-length cDNAs or 5' ESTs available for each locus (personal communication from TAIR). 

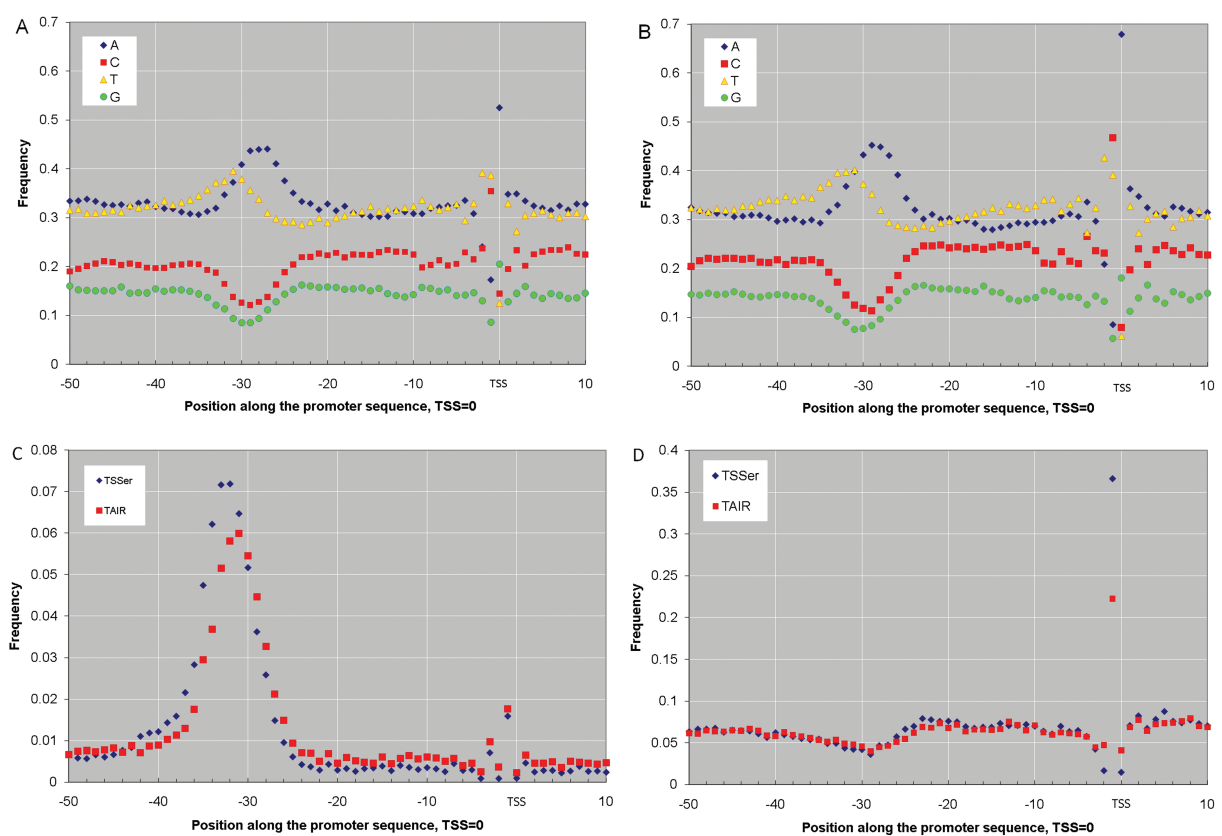

FIG. 1. Nucleotide distribution in 15,853 promoter sequences of Arabidopsis thaliana. Position 0 corresponds to the first transcribed nucleotide. (A) TSS predicted by TAIR, (B) TSS predicted by TSSer, (C) frequency of TATA, (D) frequency of CA.

\section{Microarray data sets}

We used microarray data from AtGenExpress developmental experiment (Schmid et al., 2005), abiotic stress experiments (Kilian et al., 2007; von Koskull-Doring et al., 2007), and nitrate response experiments from (Wang et al., 2003). The datasets were downloaded from the TAIR web site (Garcia-Hernandez et al., 2002) and processed with the
RMA (Robust Multichip Average) algorithm (Bolstad et al., 2003; Irizarry et al., 2003).

\section{Motif finding algorithm (Motifer)}

For every combination of gene and experimental condition, we calculated an Adaptable Expression Measure (AEM) indicating how strongly this gene is expressed under the

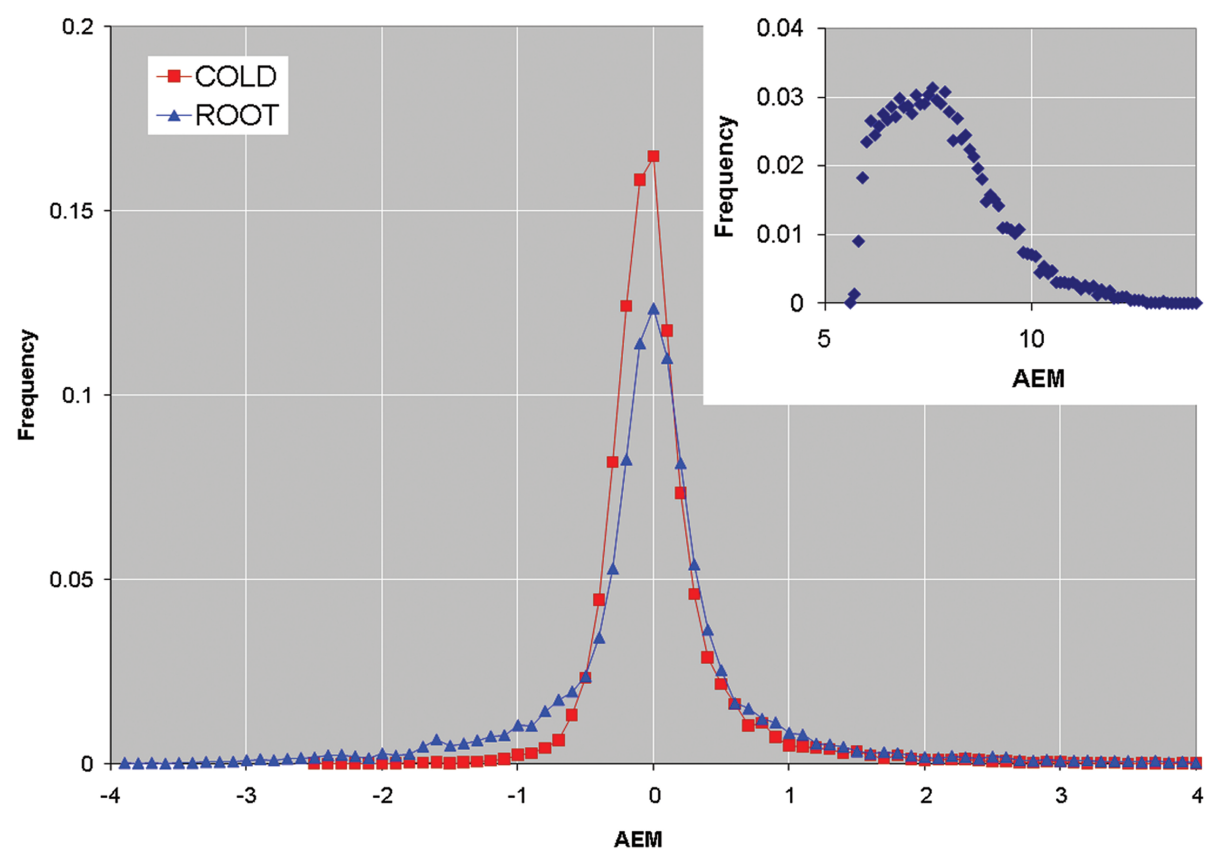

FIG. 2. Distribution of adaptable expression measure for cold, roots and strength dataset. 
given condition. For example, if we are interested in discovering cis-elements responsible for gene induction under drought, the AEM in this case is a log ratio of expression intensity under drought to expression intensity under normal conditions. The seed-specificity AEM is defined as a log ratio of expression intensity of a gene expressed in seeds to its average expression in other tissues. Promoter strength AEM is defined as an average log expression intensity, and promoter variability AEM is defined as a standard deviation of $\log$ intensities across different tissues. For every sequence motif $w$ of $l$ nucleotides in a fixed-size window around position $k$ in promoter $i$, we employ the notation of Kronecker's delta symbol in the following form:

$\delta(w, k, i)=\left\{\begin{array}{l}1, \text { if } w \text { is present in promoter } i \text { near position } k \\ 0, \text { if } w \text { is not present in promoter } i \text { near position } k\end{array}\right.$

For each motif $w$ in position $k$ we compute a sum of AEMs $e_{i}$ of promoters having this motif in a given position:

$$
d(w, k)=\sum_{i} \delta(w, k, i) \cdot e_{i}
$$

To assess significance of each motif, we compute its Z-score as:

$$
z(w, k)=\frac{d(w, k)-<e>\cdot n(w, k)}{\sigma \cdot \sqrt{n(w, k)}},
$$

where $\langle e\rangle$ is the average AEM value, $\sigma$ is a standard deviation of the expression of all promoters, and $n(w, k)$ is the number of promoters having motif $w$ near position $k$. Words with large Z-scores are the most important motifs for the target expression pattern. We validate the statistical significance of these motifs by performing a $t$-test for the expression number distribution in two sets of promoters: with the motif and without the motif. The most significant $l$-mers are further clustered together into longer motifs based on their location in promoter and sequence similarity. The clustering works as follows: if two l-letter words are located at most $x$ nucleotides apart in the promoter and the $m$-letter suffix of the first word overlaps $m$-letter prefix of the second word, they are merged into one $(2 * l-m)$ letter word. Default parameters for Motifer are: the suffix length of $\mathrm{m}=l-1$ and the distance of $x=10$ nucleotides.

We have tested two implementations of this algorithm: Motifer and Motifer Gold. Motifer is a fast algorithm that can typically obtain results for 10,000 promoters in less than $1 \mathrm{~min}$ on an Intel-based computer with $2 \mathrm{GHz}$ processor.
However, Motifer search is limited to exact words. Motifer Gold produces position-specific matrices (PSMs) that relax the sequence identity requirements and result in increased sensitivity. A PSM is constructed in the beginning from the most significant motifs and is presented as a matrix of $1 \mathrm{~s}$ (for those nucleotides which are present in the motifs) and 0s for others nucleotides. In the following iteration, a new PSM is formed from the old matrix by adding one nucleotide on each side. The PSM weights for nucleotides present in the promoter are calculated by adding AEMs of the promoters containing the motif. At the end of the iteration, when all the promoters are screened, the PSM is normalized so that the sum of all nucleotides weights in every position equals to 1 . If a terminal nucleotide is uninformative (e.g. has weight close to zero) the PSM is shortened by one nucleotide. The number of iterations can be varied; for the subsequent analysis we have used five iterations that we have found to be generally optimal (data not shown).

\section{Results and Discussion}

\section{Validation of transcription start site prediction (TSSer)}

We hypothesize that a critical component in identifying motifs is taking into account the position of a motif in relationship to the TSS. In order to do this we first need to accurately identify the TSS, for which we have constructed the application called TSSer and applied it to identify promoters of Arabidopsis thaliana. We have predicted positions of TSSs for 15,853 loci $(13,279$ of which contain at least two confirming ESTs). To validate the accuracy of this approach we examined the distribution of nucleotide content in our compilation of promoter sequences and compared the nucleotide distribution obtained from TAIR. We have extracted TAIR promoters for the 15,853 loci annotated by TSSer. The distribution of nucleotides around the start site predicted by TAIR and TSSer are shown in Figure 1A and B, respectively, revealing an AT-rich region (TATA box) around position -30 consistent with previous observations (Molina and Grotewold, 2005). We also observe a CA motif with last A being the first transcribed nucleotide in roughly $70 \%$ of all genes. Comparison of TSSs predicted by TAIR to those predicted by TSSer revealed that $37 \%$ of TSS annotations are the same between the two programs and in another $25 \%$ differ by less than 10 nucleotides. The average difference in TSS predictions is 30 nucleotides.

Table 1. Number of Sequences and Negative Logarithm of the p-Value Computed for each Expression Dataset for the Most Significant Words Found by MatrixREDUCE, Motifer, and Motifer Gold

\begin{tabular}{lcccc}
\hline Experiment & $\begin{array}{c}\text { Number of } \\
\text { promoters }\end{array}$ & $\begin{array}{c}\text { MatrixREDUCE } \\
\text {-log (p-value) }\end{array}$ & $\begin{array}{c}\text { Motifer } \\
\text {-log (p-value) }\end{array}$ & $\begin{array}{c}\text { MotiferGold } \\
\text {-log (p-value) }\end{array}$ \\
\hline 7KN03 & 9,213 & 4.95 & 13.67 & 15.67 \\
COLD & 12,742 & 2.40 & 19.35 & 40.91 \\
DROUGHT & 11,635 & 11.58 & 16.18 & 28.39 \\
FLOWERS & 9,310 & 0.70 & 19.80 & 17.70 \\
HEAT & 9,415 & 11.70 & 6.48 & 35.20 \\
LEAVES & 9,132 & 14.65 & 23.07 & 17.54 \\
ROOTS & 9,235 & 0.38 & 13.29 & 21.29 \\
SEEDS & 9,180 & 9.73 & 6.92 & 43.42 \\
STRENGTH & 11,060 & 0.55 & & 9.56 \\
\hline
\end{tabular}


Because the TATA box represents a convenient landmark for many promoter regions, we examined the frequency of this in relation to the distance from the predicted TSS. Figure $1 \mathrm{C}$ shows that the location of the TATA box has a narrower distribution for TSSer-predicted promoters. The TATA box is present from 40 to 30 nucleotides upstream of the TSS at a higher frequency for TSSer predicted promoters than in TAIR annotations ( 0.46 vs. 0.35 respectively). We also note that the peak of the TATA box distribution from TSSer is shifted slightly upstream from the predicted transcription start site. The position of this peak for TAIR promoters is closer to the TSS, possibly due to the selection of the longest $5^{\prime}$ EST as a start of transcription. Figure 1D shows the nucleotide distributions at the predicted TSS for TSSer and TAIR predictions revealing a significant difference in the frequency di-nucleotide CA at the TSS. Frequency of CA at position -1 is $37 \%$ for TSSer and $22 \%$ for TAIR. The nucleotides at the TSS also show an increased preference for TCA motif at the TSS $(0.18$ vs. 0.10$)$. TCA motif was previously described to be characteristic of the TSS (Berendzen et al., 2006). Because the TATA, CA, and TCA motifs were not used in generating the TSS prediction, the increased frequency suggests an increased accuracy in prediction by the TSSer algorithm.

Some eukaryotes have a high rate of alternative splicing associated with sophisticated organization of promoter regions and presence of alternative transcription start sites to enable refined expression patterns (for discussion see Kawaji et al., 2006; Ohler, 2006). The model plant Arabidopsis thaliana has relatively low $(\sim 7 \%)$ alternative splicing rate (Alexandrov et al., 2006). In our analysis we used the "best" TSS defined as the mode of the distribution of putative TSSs. However, we retain information about all putative TSSs, and hence, our method is suitable for analysis of more complex promotes. Our algorithm can be further improved by the incorporation of additional information, e.g. skew in CG content near the TSS (Tatarinova et al., 2003; Fujimori et al., 2005), tiling array data, and positions of known promoter motifs.

\section{Comparison between Motifer and MatrixREDUCE}

The increased accuracy of predicting the TSS enables us to search more confidently for position specific promoter elements, and this advantage was built into the Motifer and Motifer Gold algorithms. In order to gauge the accuracy of this combined method, we compared our algorithms to another similar program called MatrixREDUCE (Foat et al., 2006) that is also capable of simultaneous analysis of thousands of promoter sequences.

We used Arabidopsis promoters as a test case due to the availability of high-quality genomic annotation, large collections of 5' ESTs, as well as microarray data. Performance of motif-finding algorithms can be assessed through crossvalidation (Bussemaker et al., 2007). To accomplish this, the entire collection of Arabidopsis promoters was randomly di-

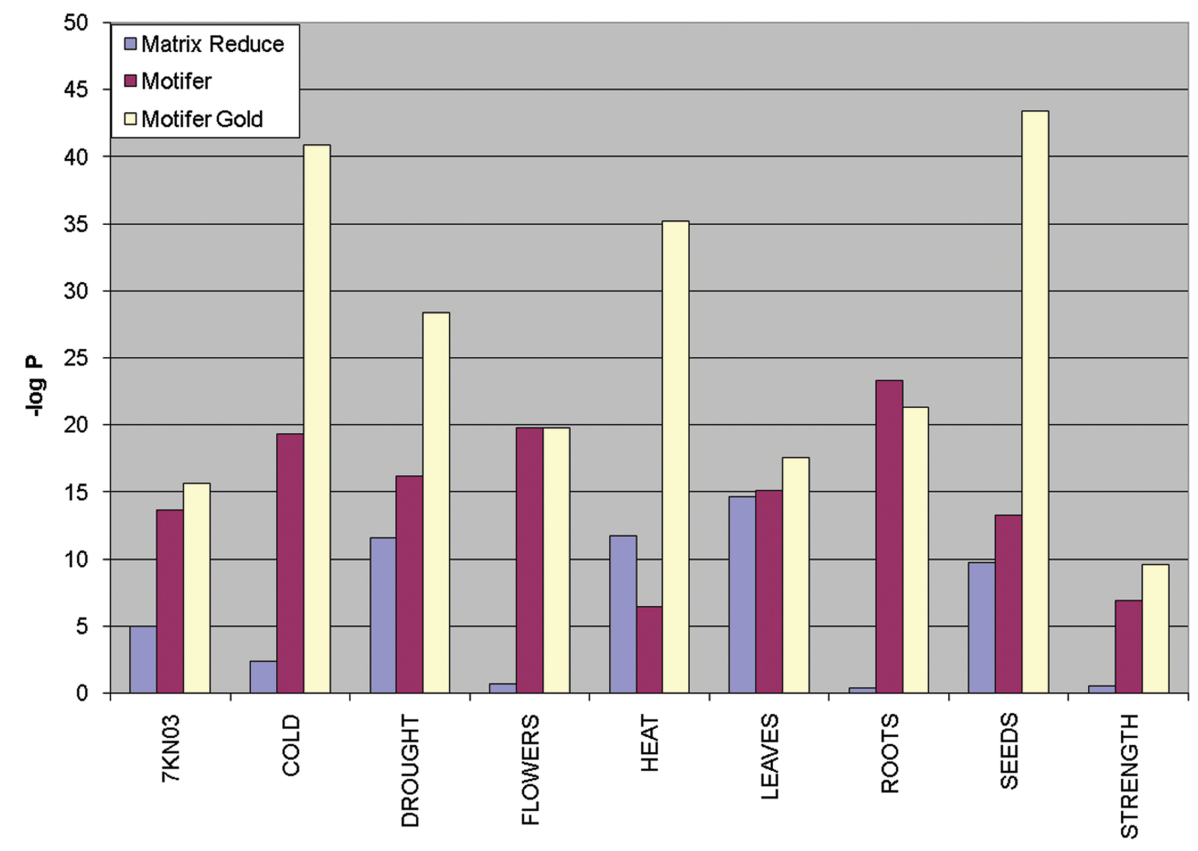

FIG. 3. Assessment of the motif effect on gene expression. The entire collection of Arabidopsis promoters was randomly divided into two subsets of the same size: one used for finding motifs (training set) and the other used for validating the effect of the found motifs on gene expression (test set). Motifer, Motifer Gold, and MatrixREDUCE were applied to nine expression datasets paired with the training promoter sequences. The highest scoring motif found by the three programs on the training set was identified for each of the nine tissues/treatments and validated on the test set. For each tissue/treatment condition the promoters were divided into those that contain the motif and those that do not. We then compared the distributions of the AEMs in these subsets. The effect of the motif presence on gene expression was assessed using $t$-test and the corresponding $p$-values were compared. Negative logarithm of the $p$-value computed for each expression dataset for the three programs is shown. 
vided into two subsets of the same size: one used for finding motifs (training set) and the other used for validating the effect of the found motifs on gene expression (test set). We examined nine microarray expression datasets for the following tissues and treatments: drought, cold, nitrogen, heat, seed, flower, root, leaf, and overall expression strength. Number of sequences for each of the datasets is shown in Table 1. For every promoter, we calculated the corresponding AEM as a characteristic expression value. Distribution of AEM in cold, root and strength datasets is shown in Figure 2.

We then applied Motifer, Motifer Gold, and MatrixREDUCE to these nine expression datasets paired with the training promoter sequences. The highest scoring motif found by the three programs on the training set was identified for each of the nine tissues/treatments and validated on the test set. For each tissue/treatment condition the promoters were divided into those that contain the motif and
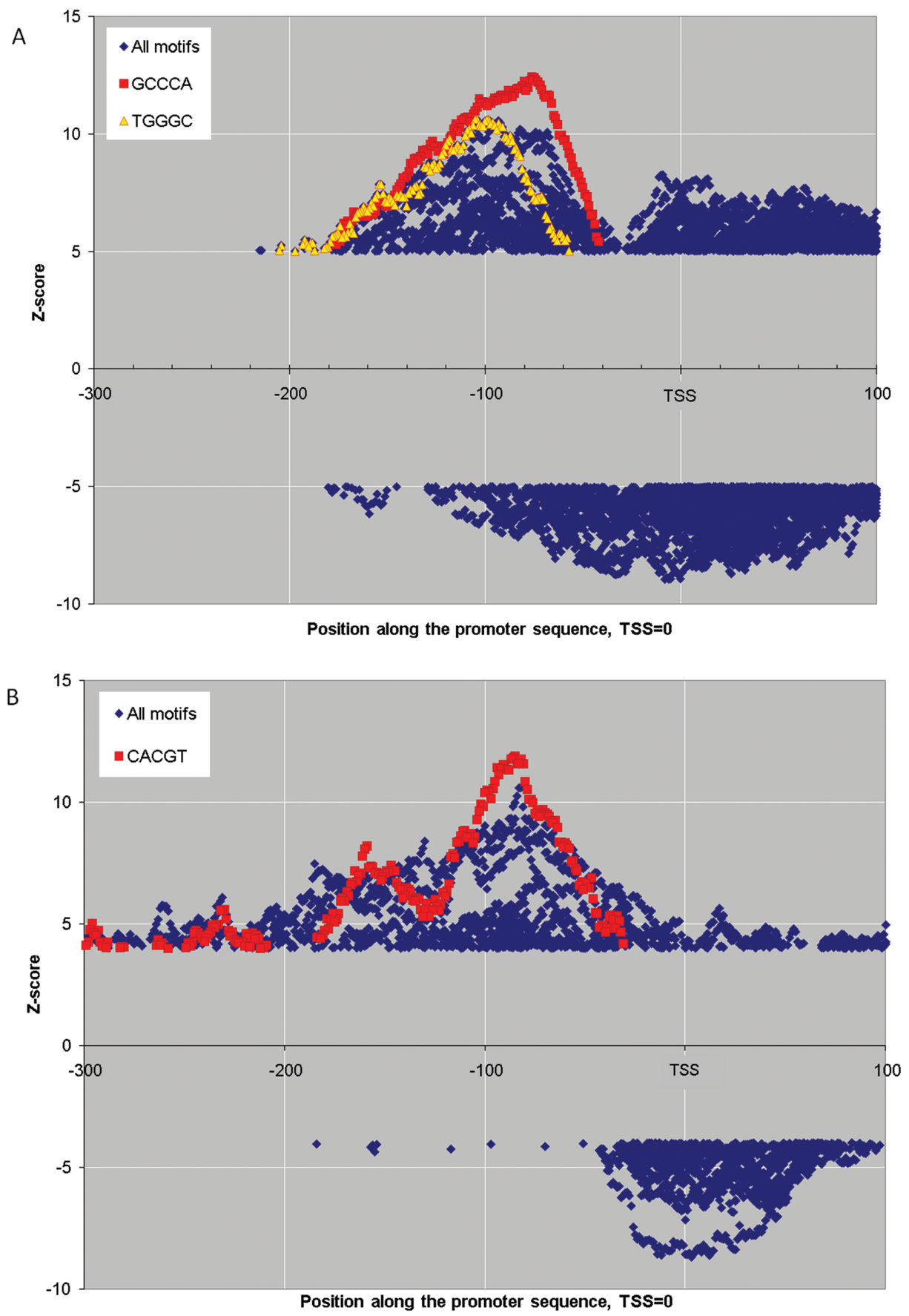

FIG. 4. Distribution of Z-scores of the most significant motifs along the promoter sequence. Positive Z-scores indicate association with transcriptional activation and negative Z-scores indicate association with transcriptional repression. (A) Motifs contributing to the overall promoter strength. (B) Motifs contributing to drought induction. (C) Motifs contributing to gene expression variability. 


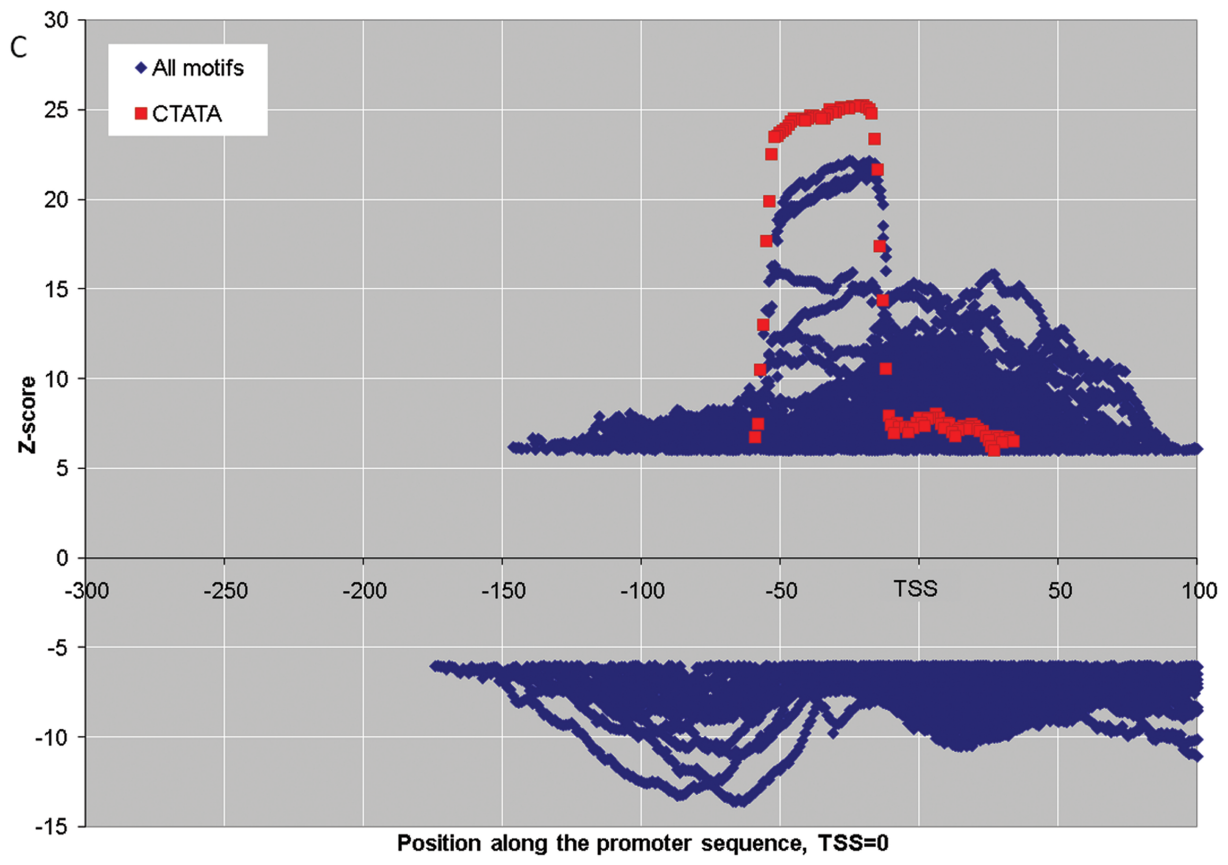

FIG. 4. (Continued).

those that do not. We then compared the distributions of the AEMs in these subsets. The effect of the motif presence on gene expression was assessed using a $t$-test, and the corresponding $p$-values were compared (Fig. 3). Overall, all three programs identified similar motifs. However, the specificity of the motifs identified through our use of positional information is higher (Fig. 3; Motifer vs. MatrixREDUCE) and the addition of a weight matrix further improves this specificity
(Fig. 3; Motifer vs. Motifer Gold). We note that MatrixREDUCE does not take into consideration positions of cis-elements with respect to TSS. These results demonstrate that significant improvement in specificity is achieved by adding positional information. We believe that this improvement reflects a biological basis and is due to similar motifs influencing expression differently due to their position relative to the TSS (Tharakaman et al., 2008).

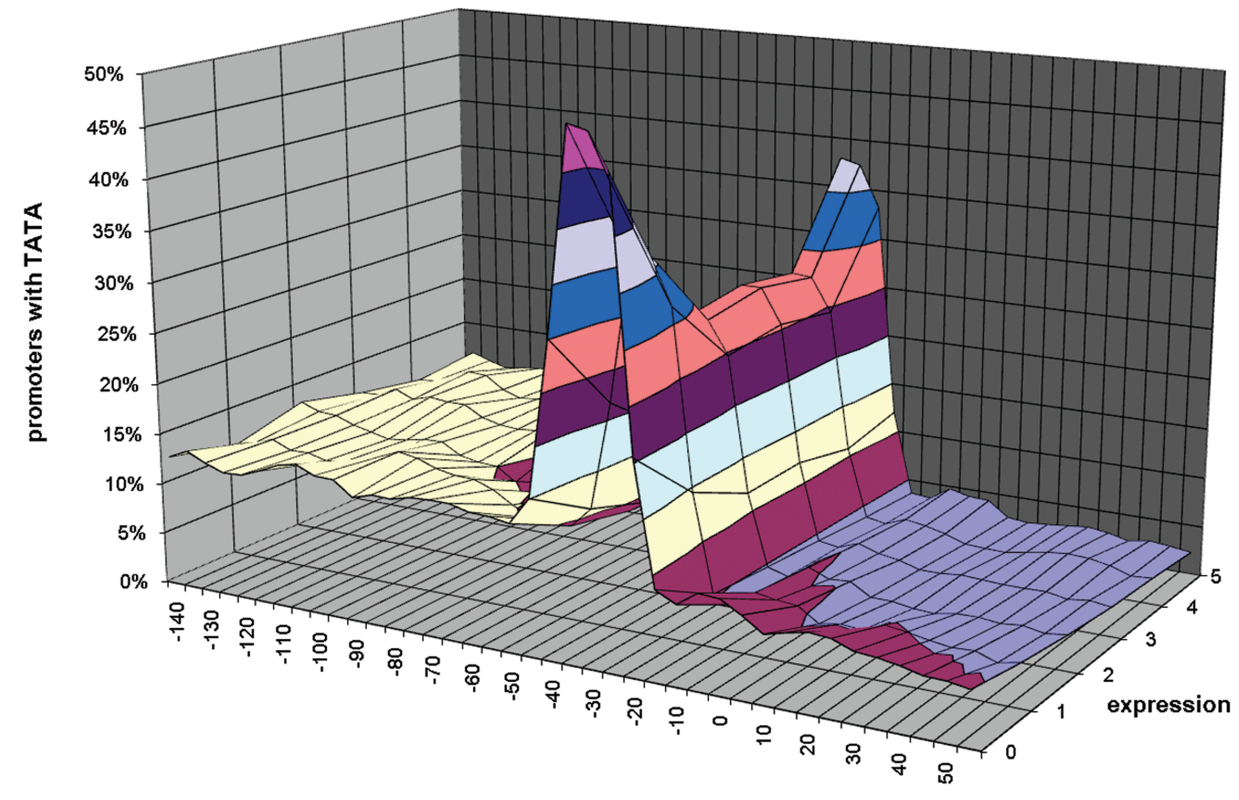

position in promoter, nt

FIG. 5. Frequency of TATA box occurrence in promoters of different strength. Expression values denote a grouping of promoters: 0 corresponds to the weakest promoters, 5 corresponds to the group of the strongest promoters 


\section{Validating Motifer on known motifs}

We examined ability of Motifer and Motifer Gold to identify previously known motifs. To find promoter motifs associated with the promoter strength, we calculated an AEM for each promoter as an average logarithm of intensity across all microarray experiments. We computed Z-scores (Eq. 2) for all "words" of five nucleotides in every position of promoter sequence. Motifs with an absolute value of Z-score larger than 4.0 are plotted against their location relative to the TSS in Figure 4A. The TATA box is considered to be an important motif for promoter strength (Molina and Grotewold, 2005); however, Figure 3A shows that no significant activating motifs (positive Z-scores) can be found around $-30 \mathrm{nt}$, a typical location of a TATA box. Repressing motifs (negative Z-scores) are more evenly distributed around the TSS. An overrepresentation of motifs GCCCA/TGGGC in core promoter regions of activated genes was previously observed by (Molina and Grotewold 2005; Rapp et al., 2001; Yamamoto et al., 2007; Yamamoto and Obokata, 2008). This motif has also been associated with dark/light inducible genes (Janaki and Joshi, 2004) and oxidative phosphorylation (Welchen and Gonzalez, 2006). We find these complimentary motifs at approximately -90 nucleotides upstream from TSS (highlighted in Fig. 4A). To test the strength of the influence on the promoter, a $t$-test was performed for the expression value difference between promoters containing or lacking the GCCCA motif. This resulted in a $p$-value of 9.3e-39, which is in agreement with previously described activating effect of these motifs (Welchen and Gonzalez, 2006). We also noticed that these two motifs tend to occur together in the promoter sequence (correlation coefficient is 0.40 ) and that the peaks of distribution between the complimentary motifs GCCCA/TGGGC are 11 and 21 bp apart, suggesting that they would be exposed on the same face of DNA when wound into a double helix.

It has also been previously reported that CACGT, a core sequence of the ABRE motif, is associated with drought response in plants (Seki et al., 2001). For this motif, we find that the $t$-test $p$-value is $5.6 \mathrm{e}-35$, indicating a strong association between this motif and expression in our drought dataset (Fig. 4B). The most important motif for seed-specific expression was CATGC (data not shown), which has previously been shown to be a part of the RY seed specific motif (Bobb et al., 1997). We also searched motifs contributing to promoter expression variability, where variability of a gene is defined as the standard deviation of its expression value across all tissues sampled in the AtGenExpress developmental experiment. It appeared that CTATA motif in position at about -30 is the most significant one with $p$-value of 3.0e-165 (Fig. 4C). Together, the identification of these previously described motifs provides confidence that the Motifer algorithm is effective at finding biologically relevant motifs.

\section{Expression of TATA+ and TATA- promoters}

In earlier analysis by Molina and Grotewold (2005), it was reported that TATA-less promoters tend to have lower expression levels based on the abundance of ESTs. To further understand the influence of TATA on gene expression, we divided all promoters of Arabidopsis thaliana into two categories: TATA+ (TATA containing promoters) and TATA(promoters without TATA). To visualize the effect of the TATA box on the average promoter expression, we divided all genes into six equally sized groups by their average expression. Promoter strength increases from group zero to group five (Fig. 5). The resulting graph demonstrates a sad-

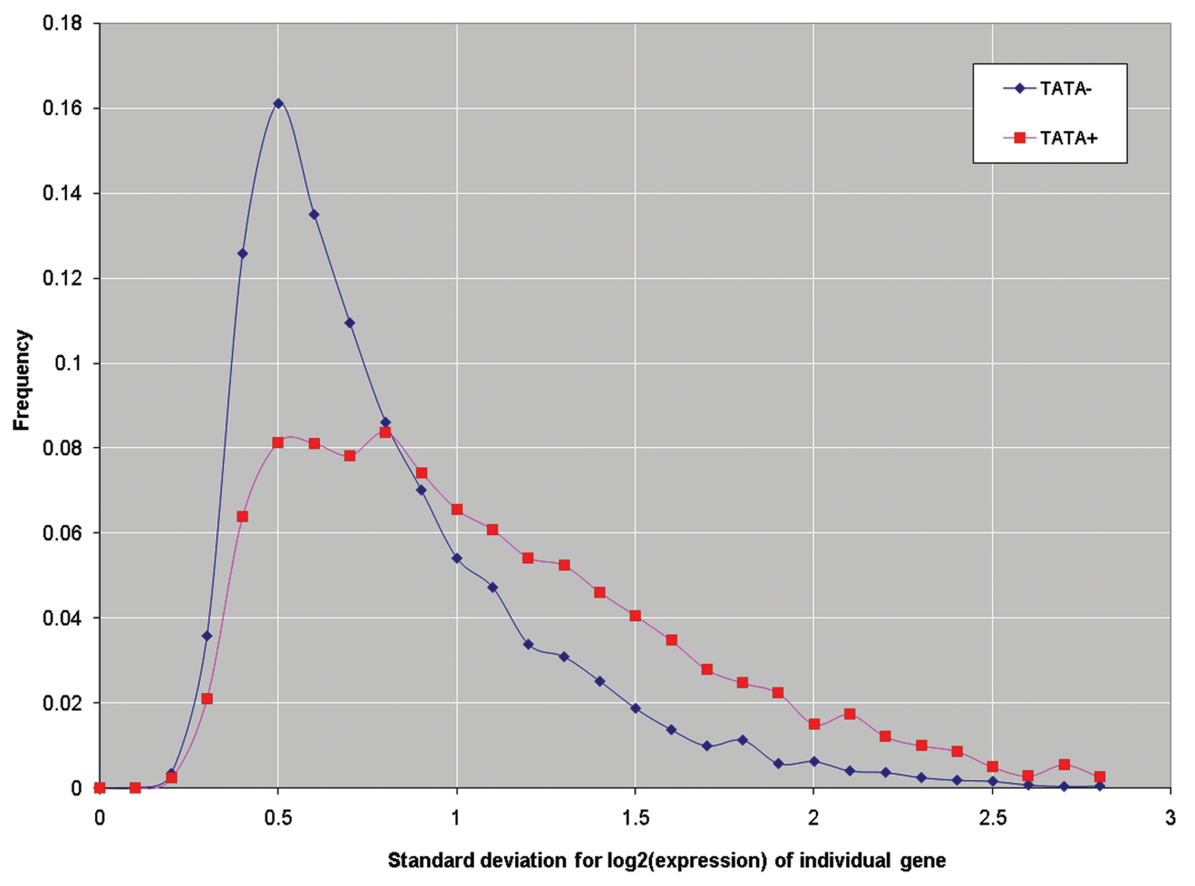

FIG. 6. Distribution of standard deviations of the log intensity values across AtGenExpress developmental experiment for TATA + and TATA - promoters. TATA + promoters show larger variability than TATA - promoters. 


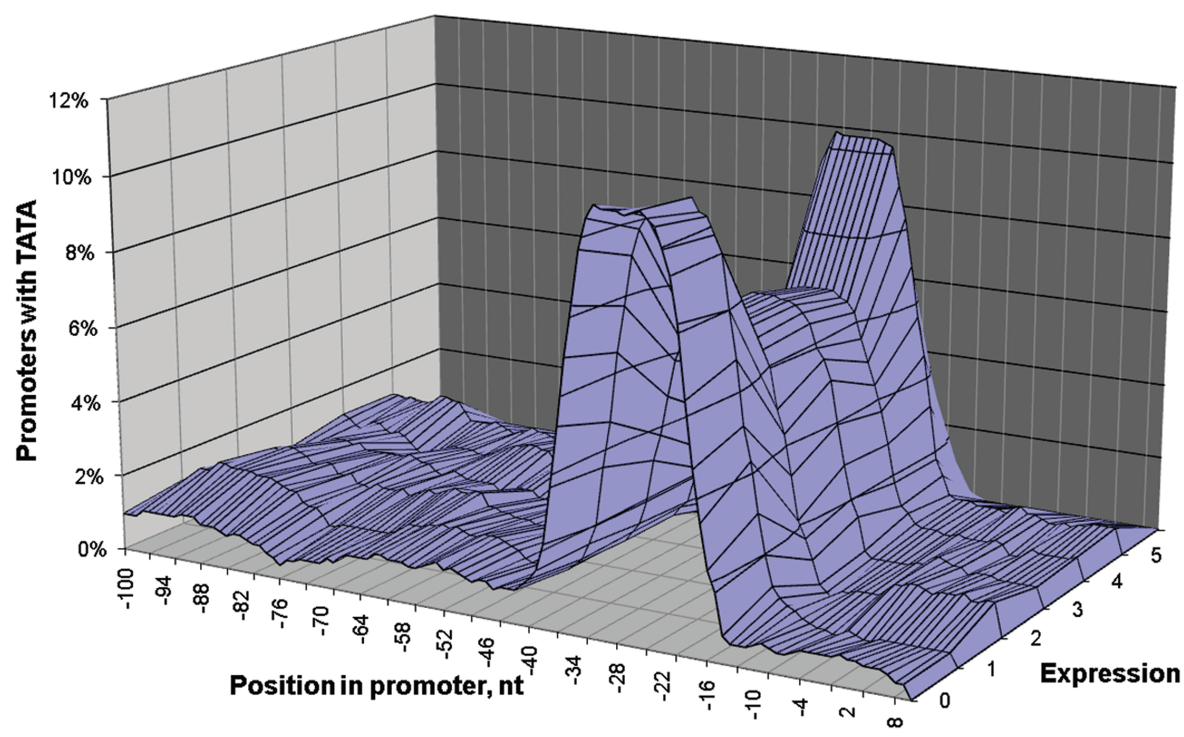

FIG. 7. Frequency of TATA box occurrence in Mus musculus promoters of different strength. Expression values denote a grouping of promoters: 0 corresponds to the weakest promoters, 5 corresponds to the group of the strongest promoters.

dle-like distribution with higher preponderance of TATA+ promoters in both weakly and strongly expressed genes but not as abundant in genes of moderate expression levels. When we searched for motifs contributing to promoter expression variability, as described in the previous section, we found that the CTATA motif in position -30 is the most significant one with $p$-value of 3.0e-165 (Fig. 4C). These observations motivated us to further investigate the influence of TATA box on gene expression variability. As a measure of variability, we used the standard deviation of log intensity values among different samples taken across different organs and developmental stages. As we can see from the resulting graph (Fig. 6), TATA+ promoters generally have larger standard deviations of microarray signal intensities, indicating higher variability of their expression. This observation indicates that the TATA box plays an important role in selective transcription regulation. Presumably, the TATA motif provides a regular structure of the TATA-binding protein (TBP)/DNA complex, which facilitates its interaction with other transcription factors. It seems logical that during

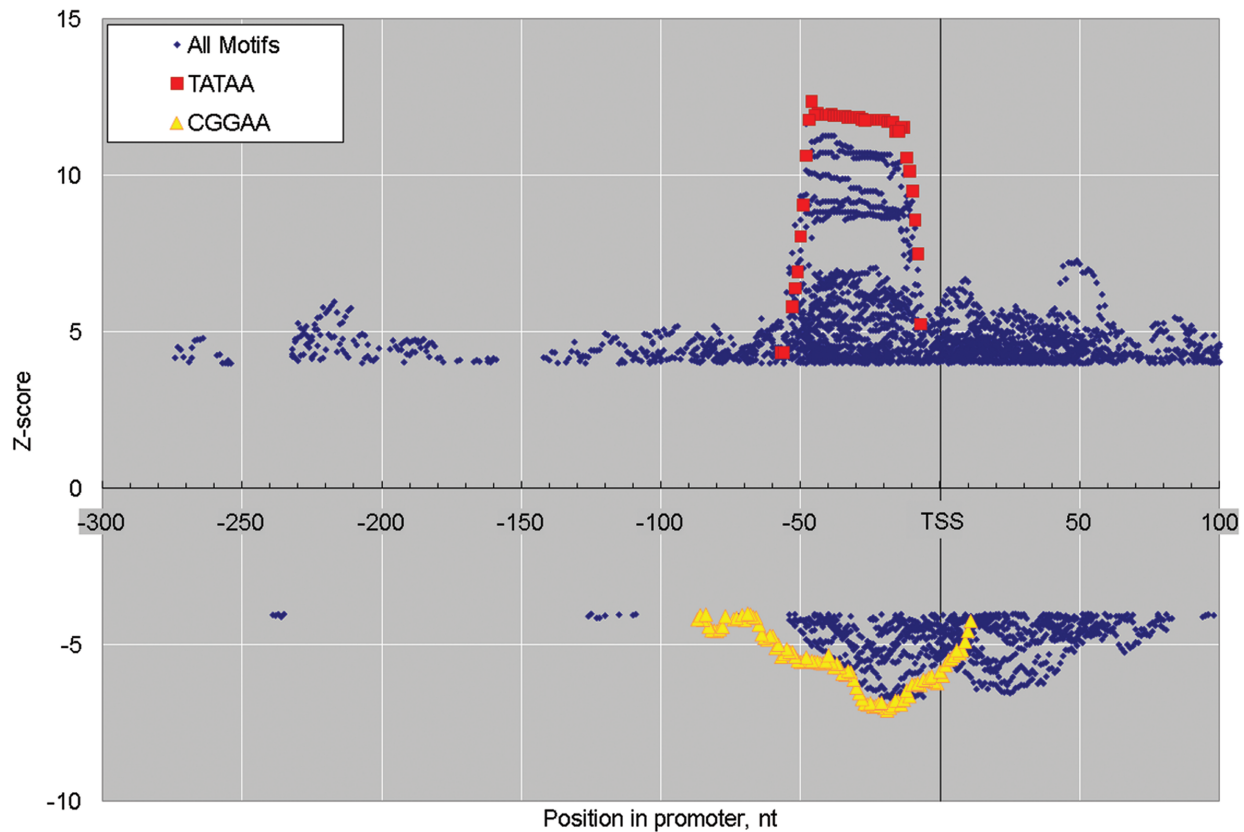

FIG. 8. Distribution of Z-scores of the most significant motifs along the Mus musculus promoter sequence contributing to the gene expression variability. Expression variability is represented as the standard deviation of log intensities across all tissues sampled in the large-scale analysis of the mouse transcriptome (Su AI, Cooke MP, et al., 2002) experiment. 
evolution the transcription factors retain the ability to interact with promoters if the TBP/DNA complex had a canonical TATA box in its core. A similar hypothesis was recently expressed by (Tirosh and Weinberger, 2006), who suggested that the presence of the TATA box in the promoter sequence contributes to the evolution of gene expression. These results agree well with findings by Basehoar et al. (2004) that, in yeast, TATA + genes are highly regulated. These results suggest that the role of the TATA-like motifs in Arabidopsis may be similar to that described in yeast.

\section{Mus musculus}

We believe that the Motifer approach is useful beyond the described plant examples and we have additionally examined promoters of the model mammal, Mus musculus. We have obtained a set of 57,944 mouse promoter sequences from the Human Genome Center (HGC), University of Tokyo (http://www.hgc.ims.u-tokyo.ac.jp/english/). We then retrieved an array of microarray measurements for 45 mouse tissues from GenBank (Su et al., 2002; experiment GSE97). Analysis of 100 nucleotides immediately upstream from the TSS shows that the frequency distribution of TATA boxes exhibits a similar saddle-like pattern (Fig. 7) to the one we have observed in the case of Arabidopsis thaliana promoters. We have applied Motifer to the analysis of expression variability of Mus musculus promoters. As before, we define expression variability as a standard deviation of log intensities across different tissues and have plotted the Z-scores above and below in Figure 8. Similar to Arabidopsis, a TATA containing motif (TATAA), located at -30 nucleotides upstream from the TSS is the most important for variability of a gene. The most prominent element that showing a strongly negative association with gene variability (displays a stable expression pattern) is the motif CGGAA, Ets response element (Koike et al., 1995). The identification of relevant mo- tifs in Mus musculus demonstrates the applicability of Motifer to organisms other than Arabidopsis.

\section{Genome ontology validation}

We were intrigued by the association of TATA motifs with variable gene expression. To understand the significance of this we have analyzed the Gene Ontology (GO) annotation of 13,279 loci of Arabidopsis thaliana. Genes were divided into five groups based on their average logarithm of expression level and counts of TATA + and TATA - were recorded for each GO category and expression group (Table 2). For each GO category that contained at least 100 genes, we have computed relative frequency Rc of TATA+/TATA - promoters according to the formula:

$$
R^{c}=\frac{T A T A_{+}^{c}}{T_{A T A}^{c}} \frac{T A T A_{-}}{T A T A_{+}},
$$

where TATA ${ }_{+}^{c}$ and TATA - are GO category-specific counts of TATA + and TATA - promoters. We note that genes participating in the stress and defense response process (e.g., response to cold, oxidative stress, abscisic acid stimulus, auxin stimulus, salt stress) tend to have higher frequency of TATA. On the other hand, genes involved with general cellular processes (e.g., those involved in embryonic development ending in seed dormancy, N-terminal protein myristoylation, signal transduction, transport, ubiquitindependent protein catabolic process, protein folding, and protein amino acid phosphorylation) had lower frequency of TATA box. Curiously, genes involved in translation scored relatively high $\left(R^{c}=1.57\right)$. This effect might be explained by observing that the ribosomal genes, which constitute the bulk of the translation-related GO category, have generally high expression levels. According to the AtGenExpress, the average expression level of 200 ribosomal genes in the developmental experiment is 11.06 on the log

Table 2. Occurrence of TATA+ and tatA - Promoters for Several Abundant Gene Ontology Categories

\begin{tabular}{|c|c|c|c|c|}
\hline Go term & $T A T A^{\mathrm{c}}+$ & TATA $A^{\mathrm{c}}-$ & $R^{\mathrm{c}}$ & p-value \\
\hline Response to oxidative stress & 71 & 55 & 2.82 & 7.0E-10 \\
\hline Response to abscisic acid stimulus & 65 & 67 & 2.12 & $6.4 \mathrm{E}-06$ \\
\hline Response to auxin stimulus & 69 & 76 & 1.98 & $1.6 \mathrm{E}-05$ \\
\hline Defense response & 67 & 74 & 1.98 & 2.9E-05 \\
\hline Response to cold & 60 & 72 & 1.82 & $3.5 \mathrm{E}-04$ \\
\hline Carbohydrate metabolic process & 81 & 101 & 1.75 & 1.3E-04 \\
\hline Translation & 125 & 174 & 1.57 & $6.4 \mathrm{E}-05$ \\
\hline Response to salt stress & 53 & 75 & 1.54 & 1.3E-02 \\
\hline Electron transport & 108 & 167 & 1.41 & 4.2E-03 \\
\hline Regulation of transcription, DNA-dependent & 160 & 276 & 1.27 & 1.3E-02 \\
\hline Proteolysis & 69 & 126 & 1.20 & 2.2E-01 \\
\hline Metabolic process & 110 & 212 & 1.13 & $2.8 \mathrm{E}-01$ \\
\hline Regulation of transcription & 90 & 209 & 0.94 & 7.1E-01 \\
\hline N-terminal protein myristoylation & 60 & 154 & 0.85 & 3.0E-01 \\
\hline Signal transduction & 30 & 83 & 0.79 & 3.1E-01 \\
\hline Transport & 60 & 166 & 0.79 & $1.5 \mathrm{E}-01$ \\
\hline Ubiquitin-dependent protein catabolic process & 32 & 111 & 0.63 & 3.0E-02 \\
\hline Protein folding & 30 & 117 & 0.56 & 4.4E-03 \\
\hline Protein amino acid phosporylation & 66 & 305 & 0.47 & 2.1E-08 \\
\hline
\end{tabular}

$\chi^{2}$ test $p$-value is used to significance of the difference between frequencies for each GO category. Counts of TATA + and TATA- , promoters in entire population are equal to 4,169 and 9,110 accordingly. 
scale, scoring above $98 \%$ of the genes of Arabidopsis thaliana. We have analyzed the frequency of TATA+ promoters across the groups of genes that have compatible expression intensity and observed that TATA box occurs less frequently in the group of translation-related genes compared to 300 highly expressed genes selected from all GO categories ( $42 \%$ vs. $58 \%$, $p$-value $<0.00002)$. A similar result was obtained by (Mencia et al., 2002) for the promoters of ribosomal proteins in yeast and later by (Tirosh and Weinberger, 2006) for promoters of ribosomal genes of yeast and humans. These associations between the TATA motif and gene groups likely to be regulated (e.g., stress response) further support the suggestion of the TATA box being associated with variable gene expression.

\section{Conclusions and Discussion}

Here we describe a method for identifying the Transcription Start Site based on Expressed Sequence Tags and genomic information (TSSer) and a novel algorithm for identifying promoter motifs (Motifer). Positional information relative to the TSS is essential for discovery of cis-elements in promoter sequences. Simultaneous analysis of a large number of promoter sequences aligned by positions of their TSSs combined with microarray data resulted in a collection of potential regulatory sequences that are statistically associated with a specific expression pattern. We applied our method to Arabidopsis thaliana and Mus musculus promoters, and identified motifs that are expected to be associated with a variety of experimental conditions. Our results are consistent with previously experimentally validated motifs. We compare performance of two implementations (Motifer and Motifer Gold) to another similar application MatrixREDUCE, and conclude that the combination of PSM representation of cis-elements and taking into account position of promoter motifs increased insight into promoter organization.

Our approach to motif discovery is based upon direct sequence features, and it is not limited to expression information, and may be applied to searching for any cis-element where positional information may be important, such as DNA structural elements. Through our examination of Mus musculus we have demonstrated the potential to use this approach in other organisms. The ability to identify multiple alternative transcription start sites is an important aspect of this work permitting extension to mammals. Other approaches have been published based on the structural largescale features of DNA, including bendability, nucleosome position, free energy and protein-DNA twist. We do not present here the results of a comparison with a recently described algorithm (EP3 from Abeel et al., 2008) because it reports a 400 nucleotide-long DNA segment, where the core promoter is likely to be located, and it does not provide the exact location or information about the direction of a promoter. However, we expect that a combination of approaches based on structural properties of promoters, direct sequence features, and experimental evidence will provide further increased accuracy. Here we also have examined the role of the TATA box in plants and mammals, and found that presence of the TATA box is associated with variable gene expression which is likely the result of more specific promoter regulation.

\section{Acknowledgments}

We are grateful to Harmen Bussemaker, Thomas Abeel, and Victor Solovyev for fruitful discussions about discovery of regulatory elements in promoters and development of the universal performance benchmark of motif-finding algorithms. We would like to acknowledge the contribution of Stanislav Freidin in preparation of the manuscripts. We thank the anonymous referees for many helpful suggestions. We acknowledge Monsanto for providing financial support for this project.

\section{Author Disclosure Statement}

The authors declare that no competing interests exist.

\section{References}

Abeel, T., Saeys, Y., Bonnet, E., Rouze, P., and Van de Peer, Y. (2008). Generic eukaryotic core promoter prediction using structural features of DNA. Genome Res 18, 310-323.

Alexandrov, N.N., Troukhan, M.E., Brover, V.V., Tatarinova, T., Flavell, R.B., and Feldmann, K.A. (2006). Features of Arabidopsis genes and genome discovered using full-length $\mathrm{CD}$ NAs. Plant Mol Biol 60, 69-85.

Argüello-Astorga, G., and Herrera-Estrella, L. (1998). Evolution of light-regulated plant promoters. Annu Rev Plant Physiol Plant Mol Biol 49, 525-555.

Bailey, T.L., and Elkan, C. (1994). Fitting a mixture model by expectation maximization to discover motifs in biopolymers. Proceedings of the Second International Conference on Intelligent Systems for Molecular Biology, pp. 28-36. (AAAI Press, Menlo Park, CA).

Basehoar, A.D., Janton, S.J., and Pugh, B.F. (2004). Identification and distinct regulation of yeast TATA box-containing genes. Cell 116, 699-709.

Benson, D.A., Karsch-Mizrachi, I., Lipman, D.J., Ostell, J., and Wheeler, D.L. (2007). GenBank. Nucleic Acids Res. 2007 35(Database issue):D21-D25.

Berendzen, K., Stuber, K., Harter, K., and Wanke, D. (2006). cisMotifs upstream of the transcription and translation initiation sites and effectively revealed by their positional disequilibrium in eukaryote genomes using frequency distribution curves. BMC Bioinformatics 7, 522.

Bobb, A.J., Chern, M. S., and Bustos, M.M. (1997). Conserved RY-repeats mediate transactivation of seed-specific promoters by the developmental regulator PvALF. Nucleic Acids Res 25, 641-647.

Bolstad, B.M., Irizarry, R. A., Astrand, M., and Speed, T.P. (2003). A comparison of normalization methods for high density oligonucleotide array data based on bias and variance. Bioinformatics 19, 185-193.

Bussemaker, H. J., Foat, B.C., and Ward, L.D. (2007). Predictive modeling of genome-wide mRNA expression: from modules to molecules. Ann Rev Biophys Biomol Struct 36, 329-347.

Down, T.A, and Hubbard, J.P. (2002). Computational detection and location of transcription start sites in mammalian genomic DNA. Genome Res 12, 458-461.

Fickett, J.W., and Hatzigeorgiou, A.G. (1997) Eukaryotic promoter recognition. Genome Res 7, 861-878.

Florea, L., Hartzell, G., Zhang, Z., Rubin, G.M., and Miller, W. (1998). A computer program for aligning a cDNA sequence with a genomic DNA sequence. Genome Res 8, 967-974. 
Foat, B.C., Houshmandi, S.S, Olivas, W.M., and Bussemaker, H.J. (2005). Profiling condition-specific, genome-wide regulation of mRNA stability in yeast. Proc Natl Acad Sci USA 102, 17675-17680.

Foat, B.C., Morozov, A.V., and Bussemaker, H.J. (2006). Statistical mechanical modeling of genome-wide transcription factor occupancy data by MatrixREDUCE. Bioinformatics 22, e141e149.

Fujimori, S., Washio, T., and Tomita, M. (2005). GC-compositional strand bias around transcription start sites in plants and fungi. 1: BMC Genomics. 28, 26.

Galas, D.J., Eggert, M., and Waterman, M.S. (1985). Rigorous pattern recognition methods for DNA sequences: analysis of promoter sequences from E. coli. J Mol Biol 186, 117-128.

Garcia-Hernandez, M., Berardini, T. Z., Chen, G., Crist, D., Doyle, A., Huala, E. et al. (2002). TAIR: a resource for integrated Arabidopsis data. Funct Integr Genomics 2, 239-253.

Ghosh, S., and Echols, H. (1972). Purification and properties of D protein: a transcription factor of Escherichia coli. Proc Natl Acad Sci USA 69, 3660-3664.

Haas, B.J., Delcher, A.L., Mount, S.M., Wortman, J.R., Smith, R.K., Jr., Hannick, L.I., et al. (2003). Improving the Arabidopsis genome annotation using maximal transcript alignment assemblies. Nucleic Acids Res 31, 5654-5666.

Hertz, G.Z., and Stormo, G.D. (1999). Identifying DNA and protein patterns with statistically significant alignments of multiple sequences. Bioinformatics 15, 563.

Hughes, J.D., Estep, P.W., Tavazoie, S., and Church, G.M. (2000). Computational identification of cis-regulatory elements associated with groups of functionally related genes in Saccharomyces cerevisiae. J Mol Biol 296, 1205-1214.

Initiative, T.A.G. (2000). Analysis of the genome sequence of the flowering plant Arabidopsis thaliana. Nature 408, 796-815.

Irizarry, R.A., Bolstad, B.M., Collin, F., Cope, L.M., Hobbs, B., et al. (2003). Summaries of affymetrix GeneChip probe level data. Nucleic Acids Res 31, e15.

Janaki, C., and Joshi, R.R. (2004). Motif detection in Arabidopsis: Correlation with gene expression data. In Silico Biol 4, 0014.

Kawaji, H., Frith, M.C., Katayama, S., Sandelin, A., Kai, C., Kawai, J., et al. (2006). Dynamic usage of transcription start sites within core promoters. Genome Biol 7, R118.

Kent, W.J. (2002). BLAT-The BLAST-like alignment tool. Genome Res 4, 656-664.

Kilian, J., Whitehead, D., Horak, J., Wanke, D., Weinl, S., Batistic, O., et al. (2007). The AtGenExpress global stress expression data set: protocols, evaluation and model data analysis of UV-B light, drought and cold stress responses. Plant J 50, 347-363.

Koike, S., Schaeffer, L., and Changeux, J.P (1995). Identification of a DNA element determining synaptic expression of the mouse acetylcholine receptor delta-subunit gene. Proc Natl Acad Sci USA 92, 10624-10628.

Mencia, M., Moqtaderi, Z., Geisberg, J.V., Kuras, L., and Struhl, K. (2002). Activator-specific recruitment of TFIID and regulation of ribosomal protein genes in yeast. Mol Cell 22, 8122-8134.

Meyersa, B.C., Kozika, A., Griegoa, A., Kuanga, H., and Michelmore, R.W (2003). Genome-wide analysis of NBS-LRR-encoding genes in Arabidopsis. Plant Cell 15, 809-834.

Molina, C., and Grotewold, E. (2005). Genome wide analysis of Arabidopsis core promoters. BMC Genomics 6, 25.

NCBI Press Releases. (2005). Public Collections of DNA and RNA Sequence Reach 100 Gigabases. http://www.nlm.nih. gov/news/press_releases/dna_rna_100_gig.html
Ohler, U., (2006) Identification of core promoter modules in Drosophila and their application to accurate TSS prediction. Nucleic Acid Res 34, 5943-5950.

Ohler, U., Liao, G., Niemann, H., and Rubin, G.M. (2002). Computational analysis of core promoters in the Drosophila genome. Genome Biol 3, 12.

Pritsker, M., Liu, Y.-C., Beer, M.A., and Tavazoie, S. (2004). Whole-genome discovery of transcription factor binding sites by network-level conservation. Genome Res 14, 99-108.

Rapp, P.W., Carlson, J.M., Michael, T.P., McClung, C.R., and Gross, R.H. (2001). Examination of Arabidopsis thaliana upstream regions for possible promoter motifs. 5th Annual Conference on Computational Genomics, TIGR Computational Genomics Presentation, Baltimore, MD; 2001.

Roth, F.R., Hughes, J.D., Estep, P.E., and Church, G.M. (1998). Finding DNA regulatory motifs within unaligned non-coding sequences clustered by whole-genome mRNA quantization. Nat Biotechnol 16, 939-945.

Schmid, M., Davison, T.S., Henz, S.R., Pape, U.J., Demar, M., Vingron, M., et al. (2005). A gene expression map of Arabidopsis thaliana development. Nat Genet 37, 501-506.

Seki, M., Narusaka, M., Abe, H., Kasuga, M., Yamaguchi-Shinozaki, K., Carninci, P., et al. (2001). Monitoring the expression pattern of 1300 Arabidopsis genes under drought and cold stresses by using a full-length cDNA microarray. Plant Cell $13,61-72$

Su, A.I., Cooke, M.P., Ching, K.A., Hakak, Y., Walker, J.R., Wiltshire, T., et al. (2002). Large-scale analysis of the human and mouse transcriptomes. Proc Natl Acad Sci USA 99, 4465-4470.

Sugahara, Y., Carninci, P., Itoh, M., Shibata, K., Konno, H., Endo, T., et al. (2001). Comparative evaluation of 5 '-end-sequence quality of clones in CAP trapper and other full-length-cDNA libraries. Gene 263, 93-102.

Suzuki, M., Koide, Y., Hattori, T., Nakamura, K., and Asahi, T. (1995). Different Sets of cis-elements contribute to the expression of a catalase gene from castor bean during seed formation and postembryonic development in transgenic tobacco. Plant Cell Physiol 36, 1067-1074.

Suzuki, Y., Yoshitomo-Nakagawa, K., Maruyama, K., Suyama, A., and Sugano, S. (1997). Construction and characterization of a full length-enriched and a 5 '-end-enriched cDNA library. Gene 200, 149-156.

TAIR, The Arabidopsis Information Resource. Available at ftp. arabidopsis.org/home/tair/Genes/TAIR7_genome_release

Tatarinova, T., Brover, V., Troukhan, M., and Alexandrov, N. (2003). Skew in CG content near the TSS in Arabidopsis thaliana. Bioinformatics 19(Suppl. 1):i313-i314.

Tharakaraman, K., Bodenreider, O., Landsman, D., Spouge, J., and Marino-Ramirez, L. (2008). The biological function of some human transcription factor binding motifs varies with position relative to the transcription start site. Nucleic Acids Res 36, 2777-2786.

Tirosh, I., and Weinberger, A. (2006). A genetic signature of interspecies variation in gene expression. Nat Genet 38.

Tompa, M., Li, N., Bailey, T.L., Church, G.M., De Moor, B., Eskin, E., et al. (2005). Assessing computational tools for the discovery of transcription factor binding sites. Nat Biotechnol 23, 137-144.

Von Koskull-Döring, P., Scharf, K.D., and Nover, L. (2007). The diversity of plant heat stress transcription factors.. Trends Plant Sci. 12, 452-457.

Wang, R., Okamoto, M., Xing, X., and Crawford, N.M., (2003). Microarray analysis of the nitrate response in Arabidopsis roots and shoots reveals over 1,000 rapidly responding genes and 
new linkages to glucose, trehalose-6-phosphate, iron, and sulfate metabolism. Plant Physiol 132, 556-567.

Welchen, E., and Gonzalez, D.H. (2006). Overrepresentation of elements recognized by TCP-domain transcription factors in the upstream regions of nuclear genes encoding components of the mitochondrial oxidative phosphorylation Machinery. Plant Physiol 141, 540-545.

Wingender, E., Chen, X., Fricke, E., Geffers, R., Hehl, R., Liebich, I., et al. (2001). The TRANSFAC system on gene expression regulation. Nucleic Acids Res 29, 281-283.

Yamada, K., Lim, J., Dale, J.M., Chen, H., Shinn, P., Palm, C.J. et al. (2003). Empirical analysis of transcriptional activity in the Arabidopsis genome. Science 302, 842-846.

Yamamoto, Y.Y., Ichida, H., Matsui, M., Obokata, J., Sakurai, T.,
Satou, M. et al. (2007). Identification of plant promoter constituents by analysis of local distribution of short sequences. BMC Genomics 8, 67.

Yamamoto, Y.Y., and Obokata, J. (2008). Ppdb: a plant promoter database. Nucleic Acids Res 36, D977-D981.

Address correspondence to: Tatiana Tatarinova Department of Mathematics Loyola Marymount University Los Angeles, CA 90045

E-mail: tatiana.tatarinova@lmu.edu 